\title{
Periodical Distribution of Discrete Gold Islands in Electrodeposition
}

\author{
S. M. Asadul Hossain* and M. Saitou
}

Department of Mechanical Systems Engineering, University of the Ryukyus, 1 Senbaru, Nishihara-cho, Okinawa, 903-0213, Japan

\begin{abstract}
Discrete gold islands formed in electrodeposition have been investigated using an island pair distribution function (IDF) as the analogy of the atomic pair distribution function (PDF) and the Fourier image analysis. An oscillatory plot in the IDF, the two halo and streak patterns in the Fourier-transformed image indicate that the gold islands have a weak periodical structure. The Monte Carlo simulation for the distribution of gold islands with no interplay or the repulsive Coulomb force well explains the origin of the periodical structure.
\end{abstract}

Keywords: Periodical structure, Pair distribution function, Fourier transformation, Repulsive Coulomb force.

\section{INTRODUCTION}

Islands formed in electrodeposition have attracted researchers owing to their scientific interests and technological importance [1-5]. Islands formed by aggregation of adatoms on a substrate affect the physical properties of the thin film because the islands that develop with time become the grains of which the thin film consists. Many research studies have explained the island growth mechanism related to the adatom formation and surface diffusion [4-9]. Considering different experimental conditions in electrodeposition, the island size and the island density are the critical parameters in thin film formation. In all cases, the discrete islands formation in a short time results in a continuous film. However, an interesting case deserves attention where the discrete islands formation exhibits oscillatory growth and eventually does not produce any film. Hence, detail analysis on the discrete islands formation will give us more understanding on island distribution and physical properties of thin film.

In order to elucidate the distribution of discrete islands, we apply the analogy of the atomic pair distribution function (PDF) that describes the density of interatomic distances in a material [10-12]. In terminology, instead of the name PDF, IDF (Island Distribution Function) is used in this study. The IDF yields the information about the distribution of gold islands and the nearest neighbor distance $\left(\mathrm{d}_{\mathrm{m}}\right)$ of islands. For example, the oscillation of IDF shows the presence of a periodical structure in the islands. The formula of IDF, G(r) becomes [10].

$G(r)=\frac{\sum_{i=1}^{N_{r}} \sum_{j=1}^{N}\left[\delta\left\{r-\left(r_{i j}\right)\right\}\right]}{N_{r}}$,

where, $N_{r}$ is the number of reference island, $N$ is the number of islands around the reference island, $r$ is the radial

*Address correspondence to this author at the Department of Mechanical Systems Engineering, University of the Ryukyus, 1 Senbaru, Nishihara-cho, Okinawa, 903-0213, Japan; Tel: +81-98-895-8635; Fax: +81-98-895-8707;

E-mail: hhossain523@gmail.com distance, $r_{i j}$ is the radial distance between the reference island (i) to another island $(j)$, and $\delta\left\{r-\left(r_{i j}\right)\right\}$ is the Kronecker delta. $G(r)$ averaged with respect to $N_{r}$. The function $G(r)$ yields the nearest neighbor distance $\left(\mathrm{d}_{\mathrm{m}}\right)$ and the property of the distribution.

In this paper, we present an experimental study on discrete gold islands formation where the islands remain as island and do not produce any film. Using the IDF (Island Distribution Function) and Fourier transformed image $[13,14]$ of the gold islands, we examine the structural distribution of the gold islands. We use the Monte Carlo simulation to examine the origin of the periodical structure by the random deposition and the repulsive Coulomb force.

This study aims at indicating (i) the distribution of the gold islands has a weak periodical structure insensitive to the substrate structure, (ii) the origin of the periodical structure of gold islands may be due to the repulsive Coulomb force.

\section{EXPERIMENTAL SETUP}

The experiment was performed using a solution of $\mathrm{HAuCl}_{4}$ of $16.67 \mathrm{~g} / \mathrm{L}$. An ITO glass ( $7 \Omega$ sheet resistances) and a carbon plate, each of which was $30 \mathrm{~mm}$ in length and $10 \mathrm{~mm}$ in width, were prepared for cathode and anode electrodes. The ITO glass in a beaker containing acetone was cleaned with an ultrasonic cleaner, rinsed with distilled water and dried. The ITO glass has a surface roughness of $2.6 \mathrm{~nm}$ and amorphous structure. To avoid any effect of grains in metal electrodes on the distribution of gold islands, the amorphous ITO glass was chosen as a substrate. The ITO glass was placed parallel to the carbon plate in an electrochemical cell at a temperature of $299 \mathrm{~K}$. The voltage of $0.6 \mathrm{~V}$ in a time range of 30 to $300 \mathrm{~s}$ was applied to the electrochemical cell with a power supply.

We use the solution of $\mathrm{HAuCl}_{4}$ with high concentration to avoid any diffusional movement of the gold ions. We choose the room temperature as many electrochemical processes involve room-temperature treatments in electrodeposition. 
Further, at fixed current density, growth does not depend on temperature.

The microscopic images of the gold islands were observed with a confocal laser scanning microscope (Keyence, VF-7500, magnification 250-2500), which measures to an accuracy to $0.01 \mu \mathrm{m}$ in height. We performed the experiment several times and obtained the similar IDF and Fourier transformed images. Hence, the reproducibility of the experiment can be ascertained.

In our preliminary experiment, to ascertain that the ITO glass plate has no preferential formation site, we performed nickel electrodeposition on the ITO glass plate. In Fig. (1), the AFM image of the nickel electrodeposit of $135 \mathrm{~nm}$ in film thickness shows that the nickel islands are approximately uniform in size and height. Hence, the ITO glass plate has no preferential formation site as it results in homogeneous formation. As other substrates such as copper, nickel or stainless steel has specified crystallographic axis which may affect the distribution of islands formed by an electrodeposition.

\section{EXPERIMENTAL RESULTS AND DISCUSSION}

The PDF usually describes the probability of finding one atom and a reference atom separated by a distance [10-12]. It is noted that the IDF defined by Eq. (1) yields the average number of islands at the distant $r$ from a reference island. In this paper, we choose the IDF as an indicator of structure in island formation.

We apply the IDF to analyze the discrete gold island distribution shown in Fig. (2a). The typical IDF plot in Fig. (2b) shows an oscillatory behavior that indicates a periodical distribution [15] of the gold islands. The presence of oscillation similar to that in the PDF plot [15] indicates a useful tool for the analysis of distribution of islands. The first peak in the PDF plot is interpreted as the nearest neighbor distance of atoms $[10,15]$. In a similar way, the first peak in Fig. (2b) indicates the nearest neighbor distance $\left(\mathrm{d}_{\mathrm{m}}\right)$ between the gold islands on average. Now, to confirm this periodical distribution of the gold islands, we analyze

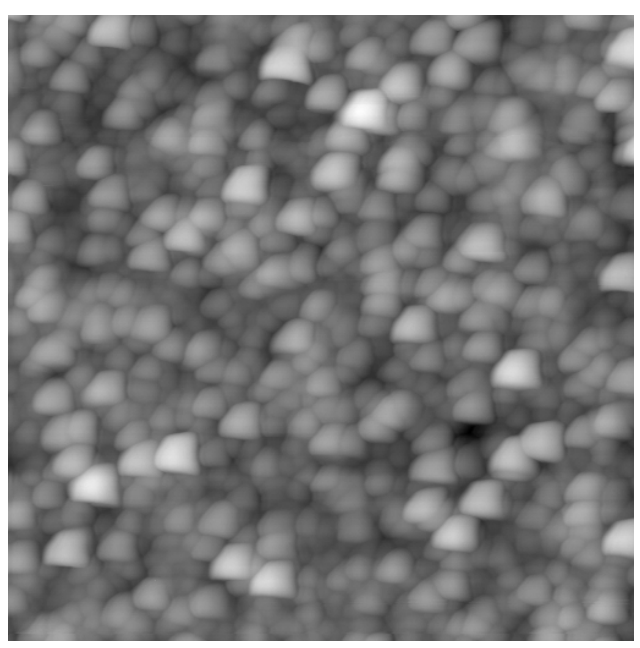

Fig. (1). AFM image of nickel electrodeposits with a size of $2 \times 2$ $\mu \mathrm{m}^{2}$ on ITO glass plate. The current density is $2 \mathrm{~mA} / \mathrm{cm}^{2}$, temp $=300 \mathrm{~K}$ and film thickness $=135 \mathrm{~nm}$. the microscopic image in Fig. (2a) with Fourier transformation. Moreover, the Fourier transformed image illustrates the periodical structure of gold islands in detail. The Fourier transformed image in Fig. (2c) supports the periodical distribution of the gold islands found in the IDF plot. This periodicity is insensitive to the preferential formation site of the ITO glass substrate. This is because the ITO glass is known to have no periodical distribution of preferential formation sites [16]. In other words, the preferential formation sites are distributed randomly.
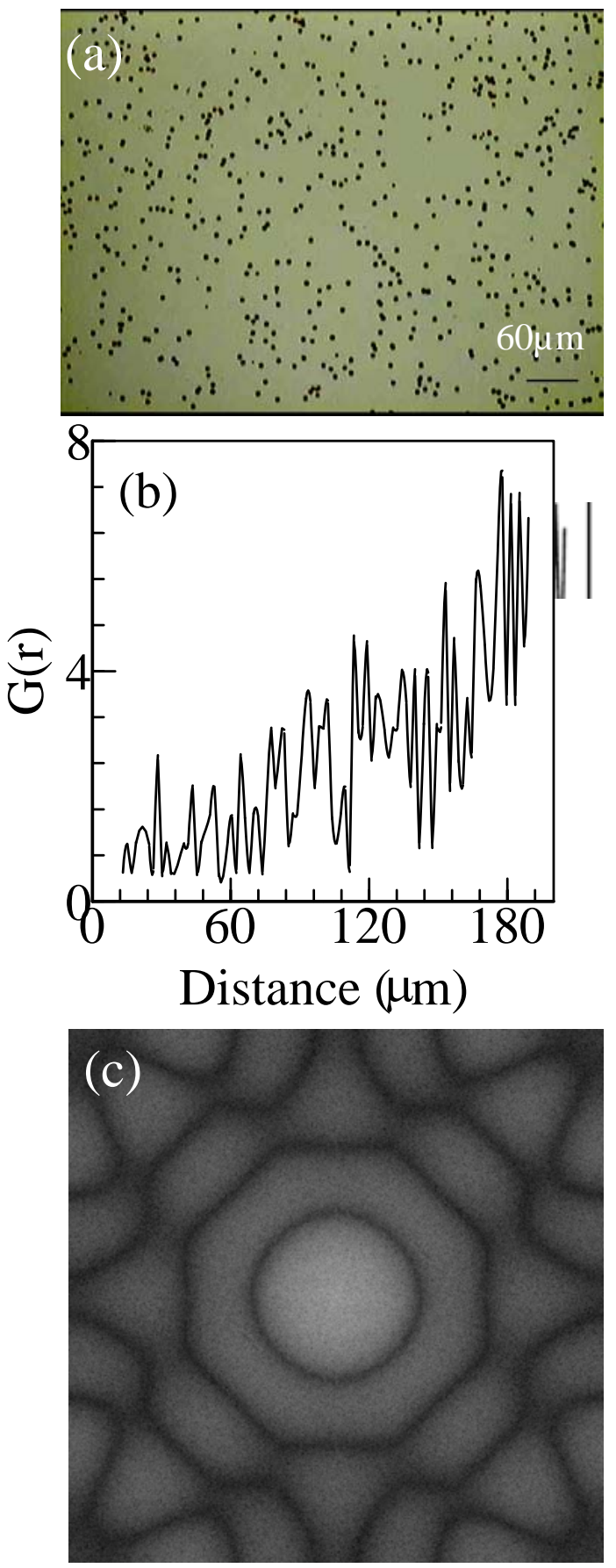

Fig. (2). a) Typical microscopic image of gold islands at deposition time $300 \mathrm{~s}$, b) Island Distribution Function (IDF) as G(r) calculated using Eq.1 from Fig. (2a), c) The Fourier transformed image of Fig. (2a) shows the presence of two halo pattern and streaks.. 
Fig. (2c) shows two halo patterns with streaks that exhibits the weak periodic distribution $[13,14]$ of gold islands as shown in the IDF plot. The two halo patterns indicate the periodical alliance of islands in Fig. (2a). The first and second halo patterns in the Fourier image correspond to the second and first peak in Fig. (2b), which shows the validity of the IDF plot. If a sharp streak line in the Fourier transform image exists, the gold islands in the real space will be aligned in a direction perpendicular to the streak line. In Fig. (2c), the two weak streak lines, which are perpendicular to each other, are observed. Therefore, in Fig. (2a) the gold islands are weakly distributed along the lines, which are perpendicular to each other. Hence, the distribution of the gold islands has a weak periodical structure.

In order to explain the origin of the periodical structure in gold islands, we use the Monte Carlo simulation [17] for deposition with no interplay between gold islands or with interplay such as the repulsive Coulomb force. Fig. (3a) shows the distribution of the random deposition of particles (no interplay) at the area $40 \times 40 \mu \mathrm{m}^{2}$ and the number of particles $\mathrm{N}=10^{5}$. The IDF plot in Fig. (3b) shows no oscillation as the experimental results in Fig. (3b). The Fourier transformed image in Fig. (3c) exhibits no halo
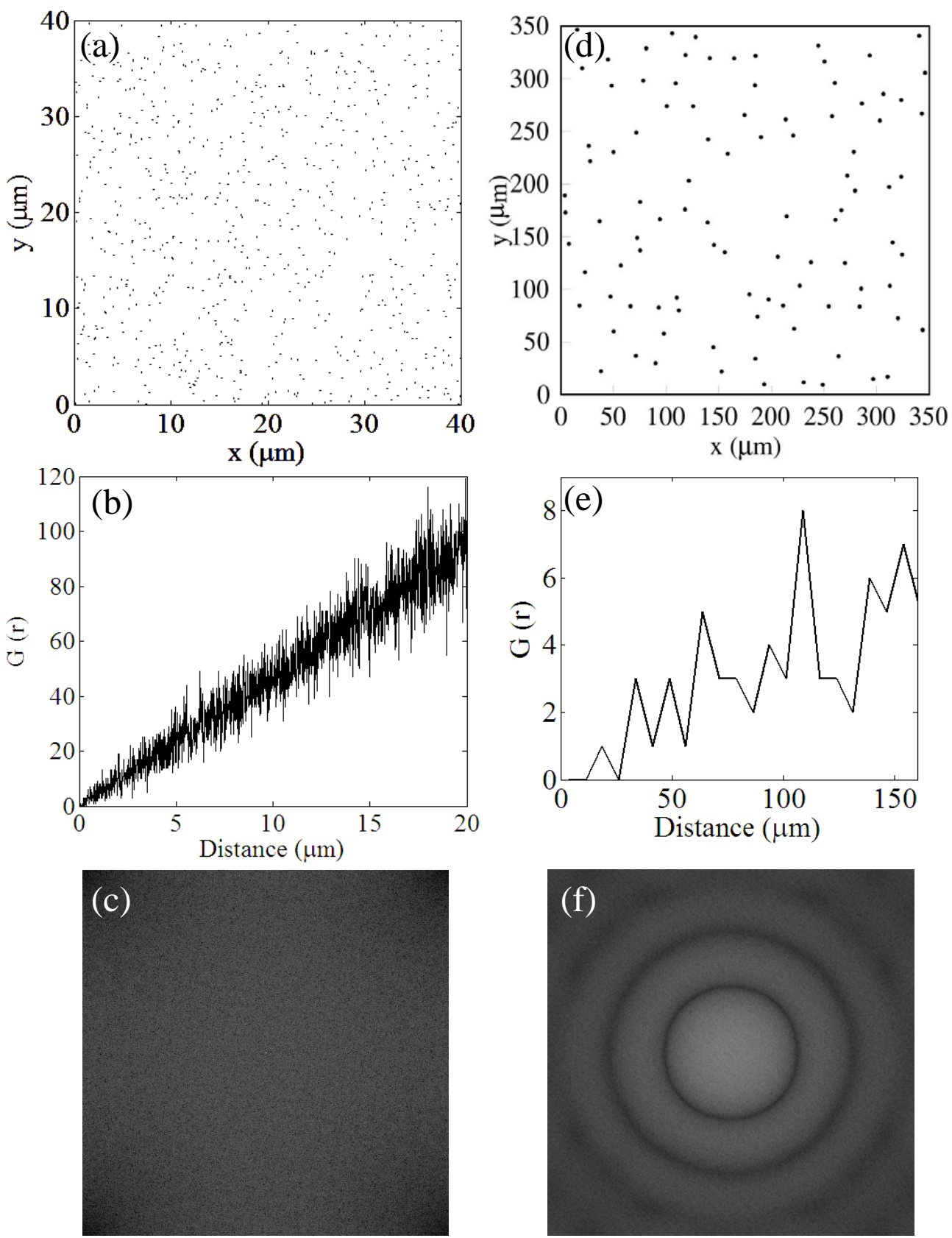

Fig. (3). Monte Carlo simulation for particle distribution a) snapshot picture in random deposition (with no interplay) at $40 \diamond 40 \mu \mathrm{m} 2$, the number of particles 105, b) IDF for Fig. (3a), c) Fourier transformed image of Fig. (3a), d) using periodic boundary condition for repulsive coulomb force (with interplay), the snapshot picture of gold particle distribution at $350 \diamond 350 \mu \mathrm{m} 2$, Monte Carlo steps 100000 and the number of particles 100, e) IDF for Fig. (3d), f) Fourier transformed image of Fig. (3d). 
pattern and streak. Hence, these calculations show that some interplay is needed to generate the periodical distribution. The gold electrodeposition from $\mathrm{HAuCl}_{4} \cdot 4 \mathrm{H}_{2} \mathrm{O}$ solution proceeds as the $\left(\mathrm{AuCl}_{4}\right)^{-}$ions transported to the ITO substrate interface by diffusion. The cathodic electrodeposition occurred as $\left(\mathrm{AuCl}_{4}\right)^{-}$ions reduced to gold atoms on ITO. We take into consideration the repulsive Coulomb force as the $\left(\mathrm{AuCl}_{4}\right)^{-}$ion and ITO substrate yield repulsive coulomb interaction at the interface. Using periodic boundary condition, the snapshot in Fig. (3d) shows the distribution of particles with the repulsive Coulomb force (i.e., interplay) at the area $350 \times 350 \mu \mathrm{m}^{2}$, Monte Carlo step 100000 and the number of particles 100. The IDF plot in Fig. (3e) exhibits an oscillatory behavior that indicates the periodical distribution. The Fourier transformed image in Fig. (3f) shows the halo patterns as same as that in Fig. (2c). Therefore, the repulsive Coulomb force between $\left(\mathrm{AuCl}_{4}\right)^{-}$ ions and ITO substrate may become the origin for the periodical structure [18].

\section{CONCLUSIONS}

In summary, the discrete gold island distribution in electrodeposition has been analyzed, using the IDF and Fourier transformed image. We present that the discrete gold island formation has a weak periodical structure insensitive to the substrate. The Monte Carlo simulation shows that the repulsive Coulomb force between $\left(\mathrm{AuCl}_{4}\right)^{-}$ions and ITO substrate may become the origin of the periodical distribution.

\section{CONFLICTS OF INTEREST}

Declared none.

\section{ACKNOWLEDGEMENT}

Declared none.

\section{REFERENCES}

[1] Barabasi, A.L.; Stanley, H. E. Fractal concepts in surface growth.; Cambridge University Press: Cambridge 1995.

[2] Guo, L.; Searson, P. C. On the influence of the nucleation overpotential on island growth in electrodeposition. Electrochim. Acta, 2010, 55, 4086-4091.
[3] Wang, K.; Niu, L.; Zong, Z.; Zhang, M.; Wang, C.; Shi, X.; Men, Y.; Zou, G. Direct electrodeposition of copper ladder structures on a silicon substrate. Cryst. Growth Des., 2008, 8, 442445.

[4] Radisic, A.; Ross, F. M.; Searson, P. C. In situ study of the growth kinetics of individual island electrodeposition of copper. J. Phys. Chem. B., 2006, 110, 7862-7868.

[5] Komsiyska, L.; Staikov, G. Electrocrystallization of $\mathrm{Au}$ nanoparticles on glassy carbon from $\mathrm{HClO}_{4}$ solution containing $\left[\mathrm{AuCl}_{4}\right]^{]}$. Electrochim. Acta, 2008, 54, 168-172.

[6] Guo, L.; Searson, P.C. Evolution of surface width in electrochemical nucleation and growth. Electrochem. Commun., 2010, 12, 431-434.

[7] Radisic, A.; Vereecken, P. M.; Searson, P. C; Ross, F. M. The morphology and nucleation kinetics of copper islands during electrodeposition. Surf. Sci., 2006, 600, 1817-1826.

[8] Martı́n, H.; Carro, P.; Creus, A. H.; Gonza'lez, S.; Salvarezza, R. C.; Arvia, A. J. Kinetics and mechanism of gold dendrite electroformation on $\mathrm{C}(0001)$. activation energy for gold adatom surface diffusion. J. Phys. Chem. B., 1999, 103, 3900-3907.

[9] Guo, L.; Searson, P. C. Anisotropic Island Growth: A New Approach to Thin Film Electrocrystallization. Langmuir, 2010, 24,10557-10559.

[10] Sun, Y. L.; Shen, J.; Valladares, A. A. Atomic structure and diffusion in Cu60Zr40 metallic liquid and glass: molecular dynamics simulations. J. Appl. Phys., 2009, 106, $0735201-0735208$.

[11] Itoh, K.; Fukunaga, T. Short-range structure of $\beta-\mathrm{V}_{2} \mathrm{D}$ : Pair distribution function analysis of x-ray and neutron diffraction. $J$. Appl. Phys., 2007, 10, 1235281-1235284.

[12] Malavasi, L.; Kim, H. J.; Proffen, T. Local and average structures of the proton conducting Y-doped $\mathrm{BaCeO}_{3}$ from neutron diffraction and neutron pair distribution function analysis. J. Appl. Phys., 2009, 105, 1235191-1235199.

[13] Saitou, M.; Fukuoka, Y. An experimental study on stripe pattern formation of Ag-Sb electrodeposits. J. Phys. Chem. B., 2004, 108, 5380-5385.

[14] Saitou, M.; Fukuoka, Y. Stripe pattern formation in Ag-Sb coelectrodeposition. Electrochim. Acta, 2005, 50, 5044-5049.

[15] Saitou, M.; Okudaira, Y.; Oshikawa, W. Amorphous structure and kinetics of phosphorous incorporation in Electrodeposited Ni-P thin films. J. Electrochem. Soc., 2003, 150, 140-143.

[16] Gobel, H. D.; Horber, J. K. H.; Gerber, Ch.; Leitner, A.; Hansch, T. W. Molecular structures of lipid monolayers on ITO glass and on graphite imaged by an STM. Ultramicroscopy, 1992, 42, 1260-1268.

[17] Sadus, R.J. Molecular Simulation of Fluids: Theory, Algorithm and Object-Orientation: Elsevier, Amsterdam, 1999.

[18] Menon, V.B.; Nikolov, A.D.; Wasan, D.T. Interfacial effects in solids-stabilized Emulsions. J. Colloid. Interface. Sci., 1988, 124, 317-327. 